\title{
SCOTROC 2B: feasibility of carboplatin followed by docetaxel or docetaxel-irinotecan as first-line therapy for ovarian cancer
}

\begin{abstract}
AR Clamp', J Mäenpää2 ${ }^{2}$ D Cruickshank ${ }^{3}$, J Ledermann $^{4}$, PM Wilkinson $^{5}$, R Welch $^{5}$, S Chan $^{6}$, P Vasey $^{7}$, B Sorbe $^{8}$, A Hindley ${ }^{9}$ and GC Jayson*, for the Scottish Gynaecological Cancer Trials Group

'Cancer Research UK Department of Medical Oncology, Christie Hospital, Manchester M20 4BX, UK; '2Department of Obstetrics and Gynaecology, Tampere University Hospital, FIN-3352I Tampere, Finland; ${ }^{3}$ Women and Children's Directorate, James Cook University Hospital, Middlesbrough TS4 3BW, UK; ${ }^{4}$ Department of Oncology, University College London, London WIP 8BT, UK; ${ }^{5}$ Department of Clinical Oncology, Christie Hospital, Manchester M20 4BX, UK; ${ }^{6}$ Nottingham City Hospital, Nottingham NG5 IPB, UK; ${ }^{7}$ Division of Oncology, Cancer Care Services, Royal Brisbane and Women's Hospital, Herston, Queensland 4029, Australia; ${ }^{8}$ Department of Gynecological Oncology, Örebro University Hospital, SE-70I 85 Örebro, Sweden;

${ }^{9}$ Rosemere Cancer Centre, Royal Preston Hospital, Fullwood, Preston PR2 9HT, UK
\end{abstract}

The feasibility of combination irinotecan, carboplatin and docetaxel chemotherapy as first-line treatment for advanced epithelial ovarian carcinoma was assessed. One hundred patients were randomised to receive four 3-weekly cycles of carboplatin (area under

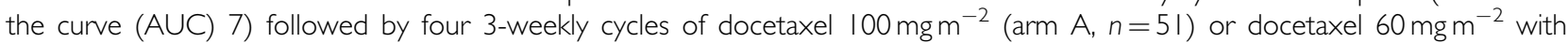
irinotecan $200 \mathrm{mg} \mathrm{m}^{-2}$ (arm B, $n=49$ ). Neither arm met the formal feasibility criterion of an eight-cycle treatment completion rate that was statistically greater than 60\% (arm A 71\% (90\% confidence interval (Cl) 58-81\%; P=0.079; arm B 67\% (90\% Cl 55-78\%; $P=0.184)$ ). Median-dose intensities were $>85 \%$ of planned dose for all agents. In arms $A$ and $B, 15.6$ and $12.2 \%$ of patients, respectively, withdrew owing to treatment-related toxicity. Grade 3-4 sensory neurotoxicity was more common in arm A (I.9 vs $0 \%$ ) and grade 3-4 diarrhoea was more common in arm B (0.6 vs 3.5\%). Of patients with radiologically evaluable disease at baseline, 50 and $48 \%$ responded to therapy in arms A and B, respectively; at median 17.1 months' follow-up, median progression-free survival was 17.1 and 15.9 months, respectively. Although both arms just failed to meet the formal statistical feasibility criteria, the observed completion rates of around $70 \%$ were reasonable. The addition of irinotecan to first-line carboplatin and docetaxel chemotherapy was generally well tolerated although associated with increased gastrointestinal toxicity. Further exploratory studies of topoisomerase-I inhibitors in this setting may be warranted.

British Journal of Cancer (2006) 94, 55-6I. doi: I0.1038/sj.bjc.66029l0 www.bjcancer.com

Published online 13 December 2005

(c) 2006 Cancer Research UK

Keywords: taxane; docetaxel; carboplatin; irinotecan; ovarian; cancer

Ovarian cancer is the fourth most commonly occurring cancer in women, with most patients presenting with extra-pelvic disease spread. Treatment of this advanced disease (International Federation of Gynecologic Oncology (FIGO) stage III-IV) involves cytoreductive surgery followed by chemotherapy. Based on the results of the pivotal GOG-111 and OV-10 studies, which demonstrated a survival advantage for cisplatin-paclitaxel over cisplatin-cyclophosphamide (McGuire et al, 1996; Piccart et al, 2000), first-line chemotherapy usually includes a platinum agent plus paclitaxel. However, median survival in these studies was only 3 years and an estimated 130000 deaths per year still occur from ovarian cancer worldwide (Shibuya et al, 2002). Paclitaxel

*Correspondence: Dr GC Jayson, Cancer Research UK Department of Medical Oncology, Christie Hospital NHS Trust, Wilmslow Road, Withington, Manchester M20 4BX, UK;

E-mail: Gordon.Jayson@christie-tr.nwest.nhs.uk

Revised II November 2005; accepted I 8 November 2005; published online 13 December 2005 treatment is also associated with peripheral neuropathy (Guastalla and Dieras, 2003). Notably, the second-generation taxane docetaxel in combination with carboplatin is as effective as carboplatinpaclitaxel for first-line therapy (Vasey et al, 2004), with myelosuppression rather than peripheral neuropathy being the predominant toxicity.

One method for improving outcomes may be to incorporate a third cytotoxic agent into first-line therapy - specifically, a new potentially non-cross-resistant agent with demonstrated activity in relapsed disease. Promising agents include the topoisomerase-I inhibitors topotecan and irinotecan, which are active in platinumresistant ovarian cancer (Takeuchi et al, 1991; Gordon et al, 2001; Bodurka et al, 2003). Irinotecan has also exhibited activity in combination with docetaxel at a recommended 3-weekly dose of irinotecan $200 \mathrm{mg} \mathrm{m}^{-2}$ and docetaxel $60 \mathrm{mg} \mathrm{m}^{-2}$ (Mäenpää et al, 1998). A response rate of $63 \%$ was reported with docetaxelirinotecan treatment for recurrent disease (Mäenpää et al, 2002); the predominant toxicities were neutropenia and diarrhoea (Mäenpää et al, 1998, 2002).

The use of triplet cytotoxic combinations, however, can be complicated by significant toxicity - in particular, myelosuppression, 
which impacts on dose intensity. One means of circumventing this is to administer agents sequentially, as has been performed successfully in the adjuvant treatment of breast cancer (Bonadonna et al, 1995). The results of the GOG-132 study suggest that such an approach could also be used in ovarian cancer (Muggia et al, 2000). In that trial, chemonaïve patients with advanced ovarian cancer received either combination cisplatin-paclitaxel or each of these drugs alone. However, as many patients receiving singleagent therapy were subsequently crossed-over to the other drug before clinical evidence of disease progression, they were essentially receiving sequential therapy. No survival differences were detected between the three arms, suggesting that sequential therapy is as effective as the concurrent approach.

Laboratory data also indicate that sequential therapy may be appropriate using platinum and taxane drugs. For instance, cell lines with p53 mutations are hypersensitive to paclitaxel but resistant to platinum, whereas cells with normal p53 function are platinum sensitive (Wahl et al, 1996). Hence, initial platinum treatment might eradicate a population of wild-type p53 tumour cells, leaving a population of predominantly mutant p53 cells that are amenable to subsequent treatment with taxanes. Thus, sequential administration of optimal doses of individual agents may allow maximum impact on distinct chemosensitive cell populations, while avoiding the toxicity problems commonly associated with concurrent combination chemotherapy.

This study investigated the feasibility of sequential carboplatin followed by docetaxel or docetaxel-irinotecan as first-line therapy in patients with FIGO stage Ic - IV epithelial ovarian, fallopian tube or primary peritoneal carcinomas.

\section{PATIENTS AND METHODS}

\section{Patient population}

Eligible patients were women aged $\geqslant 18$ years with a histopathological diagnosis of invasive epithelial ovarian carcinoma (EOC), fallopian tube cancer or ovarian-type primary peritoneal cancer. Other inclusion criteria were as follows: FIGO disease stage Ic-IV (stage Ic patients were limited to those with malignant cells in ascitic fluid, peritoneal washings or with tumour on the surface of the ovary); Eastern Cooperative Oncology Group performance status of $0-2$; no prior chemotherapy or radiotherapy; adequate bone marrow (neutrophils $>1.5 \times 10^{9} 1^{-1}$ or platelets $>100 \times 10^{9} 1^{-1}$, haemoglobin $>9.0 \mathrm{~g} \mathrm{dl}^{-1}$ ), renal (serum creatinine $<1.25 \times$ upper limit of normal (ULN)) and hepatic function (bilirubin $<1 \times$ ULN, aspartate aminotransferase/alanine aminotransferase $<1.5 \times$ ULN and alkaline phosphatase $<2.5 \times$ ULN).

Exclusion criteria included the following: borderline or mixed mesodermal tumours; previous or concurrent malignancy within the preceding 5 years (except curatively treated uterine cervical carcinoma in situ or basal cell skin carcinoma); concurrent severe and/or uncontrolled comorbidities; prior serious allergic reactions; ongoing bowel obstruction or current inflammatory bowel disease or chronic diarrhoea; pregnancy or lactation; symptomatic peripheral neuropathy $\geqslant$ grade 2 . A maximum of 8 weeks was permitted between initial surgery and starting trial chemotherapy.
All patients gave written informed consent. The trial was approved by the Manchester Ethics Committee and by the local ethics committees of the other participating hospitals.

\section{Treatment}

All patients initially received four cycles of carboplatin AUC 7 (AUC $=$ area under the curve, calculated using the Calvert formula (Calvert et al, 1989) where the glomerular filtration rate (GFR) was measured using an isotopic method). Carboplatin was infused over $1 \mathrm{~h}$ in $500 \mathrm{ml}$ of $5 \%$ dextrose every 3 weeks. After carboplatin initiation treatment, arm A patients received docetaxel $100 \mathrm{mg} \mathrm{m}^{-2}$ (1-h infusion) every 3 weeks for four cycles and arm B patients received docetaxel $60 \mathrm{mg} \mathrm{m}^{-2}$ (1-h infusion) followed $\geqslant 30 \mathrm{~min}$ later by irinotecan $200 \mathrm{mg} \mathrm{m}^{-2}$ (30-min infusion) every 3 weeks for four cycles.

Docetaxel premedication comprised $8 \mathrm{mg}$ oral dexamethasone twice daily for 3 days, commencing $24 \mathrm{~h}$ before docetaxel administration in both arms. No specific premedication for irinotecan was given. If symptoms suggestive of an acute cholinergic syndrome developed during treatment, atropine ( $0.25 \mathrm{mg}$ subcutaneous) was recommended and given prophylactically with subsequent cycles. High-dose loperamide was administered for delayed diarrhoea and fluoroquinolone antibiotics if diarrhoea occurred in the context of fever or grade 3-4 neutropenia.

\section{Dose and schedule modification}

On each scheduled treatment day, full doses of all drug(s) were administered only if neutrophils were $\geqslant 1.5 \times 10^{9} 1^{-1}$ and platelets $\geqslant 100 \times 10^{9} 1^{-1}$; otherwise, a treatment delay of $\leq 2$ weeks was permissible. Haematological recovery after 1 week resulted in the full dose being given at the next treatment cycle. If treatment was delayed by $>1$ week, or was complicated by neutropenic fever or grade 4 thrombocytopenia, the dose was reduced to dose level -1 (Table 1). Recurrence of significant haematological toxicity with subsequent cycles resulted in a further reduction to dose level -2 . A delay of $>2$ weeks for haematological recovery or further significant haematological toxicity after two dose reductions necessitated patient withdrawal from the study.

Prophylactic oral antibiotics were administered with subsequent cycles in patients with grade 4 neutropenia accompanied by a single oral temperature of $>38.5^{\circ} \mathrm{C}$. Routine use of haematopoietic growth factors was not recommended. If serum creatinine increased to $>1.25 \times$ ULN with carboplatin, the GFR was remeasured. A fall in GFR of $>25 \%$ from baseline resulted in patient withdrawal from protocol therapy. The development of grade 3-4 mucositis or hepatic toxicity during docetaxel chemotherapy necessitated treatment delay until resolution of toxicity to $<$ grade 2 and subsequent cycles were administered at dose level -1 .

For patients receiving irinotecan, the occurrence of grade 3-4 diarrhoea (despite appropriate management) led to a $25 \%$ dose reduction of irinotecan with subsequent cycles. If significant diarrhoea recurred, the docetaxel dose was also reduced by $25 \%$ and treatment was discontinued if this toxicity still persisted. All

Table I Dose levels after reduction for toxicity

\begin{tabular}{lccc}
\hline Study arm & Dose level $\mathbf{0}$ & Dose level I & Dose level 2 \\
\hline Carboplatin & AUC 7 & AUC 6 & AUC 5 \\
Docetaxel (arm A) & $100 \mathrm{mg} \mathrm{m}^{-2}$ & $75 \mathrm{mg} \mathrm{m}^{-2}$ & $60 \mathrm{mg} \mathrm{m}^{-2}$ \\
Docetaxel-irinotecan (arm B) & Docetaxel: $60 \mathrm{mg} \mathrm{m}^{-2}$; irinotecan: $200 \mathrm{mg} \mathrm{m}^{-2}$ & $25 \%$ reduction with both drugs & Further $25 \%$ reduction with both drugs \\
\hline
\end{tabular}

$\mathrm{AUC}=$ area under the curve. 
patients experiencing grade 3-4 neurotoxicity or significant cardiac toxicity were withdrawn from treatment.

\section{Clinical assessments}

A full clinical examination (including pelvic examination) and electrocardiography were performed at baseline. Clinical examination was repeated before each treatment cycle. Laboratory investigations at baseline incorporated a full blood count (FBC; including differential white cell count), full biochemical profile and CA-125. The FBC and full biochemical profile were repeated on the day of chemotherapy administration during carboplatin chemotherapy, and weekly during docetaxel-based chemotherapy. CA-125 measurement was repeated with each treatment cycle.

Disease extent at baseline was established by abdominopelvic computed tomography (CT) scan and chest radiograph. Radiological assessment was repeated after cycles 4 and 8 if evaluable disease was present on baseline imaging or if there was clinical or CA-125 evidence of disease progression. If interval debulking was performed after carboplatin chemotherapy, repeat imaging was required before starting docetaxel-containing chemotherapy. Disease response was assessed using modified Southwest Oncology Group criteria. Patients with disease progression after completing the carboplatin phase proceeded to the docetaxel-based treatment phase (unless the treating clinician deemed this inappropriate).

Patient quality of life (QoL) was assessed at baseline using the European Organisation for Research and Treatment of Cancer (EORTC) core questionnaire QLQ-C30 combined with ovarian cancer module QLQ-OV28. These questionnaires were repeated after completion of carboplatin treatment and before each docetaxel-based chemotherapy cycle. Toxicities were documented throughout chemotherapy using the National Cancer Institute of Canada Expanded Common Toxicity Criteria (version 2.0).

Patient follow-up - including full clinical and pelvic examinations, serum CA-125 measurement and QoL assessment - was performed every 2 months until disease progression or for a maximum of 2 years. Abdominopelvic CT scan was performed if progressive disease was suspected.

\section{Statistical considerations}

This was a multicentre, prospective, two-arm, randomised phase II feasibility study. Treatment allocation was performed by minimisation with stratification for centre, FIGO stage, performance status and residual disease. The primary end point was the percentage of patients completing eight cycles of chemotherapy: $80 \%$ was deemed to be clearly acceptable, $60-80 \%$ a 'grey area' and $<60 \%$ clearly unacceptable. The study was designed to test the null hypothesis that the completion rate was $\leqslant 60 \%$ against the alternative of $>60 \%$ using Fleming's test (Fleming, 1982). Setting a one-sided significance level of $5 \%$ and a power of $90 \%$ for a true completion rate of $80 \%$ required 44 patients to be recruited to each arm.

The study was not powered to detect differences in efficacy between the three arms; randomisation was utilised to ensure that patients with similar characteristics entered each arm. However, it also permitted a preliminary analysis to be made of the range of efficacy to be expected.

Exploratory comparisons of patient characteristics and treatment outcomes between the two arms were carried out using the $\chi^{2}$ test and Fisher's exact test.

\section{RESULTS}

\section{Patients}

Between June 2001 and September 2002, 100 patients (51 in arm A, 49 in arm B) were recruited from 17 European centres.
Table 2 Patient demographics

\begin{tabular}{|c|c|c|c|c|}
\hline Age & \multicolumn{2}{|c|}{ Arm A $(n=5 I)$} & \multicolumn{2}{|c|}{ Arm B $(n=49)$} \\
\hline $\begin{array}{l}\text { Median } \\
\text { Range }\end{array}$ & \multicolumn{2}{|l|}{$\begin{array}{c}56 \\
31-72\end{array}$} & \multicolumn{2}{|l|}{$\begin{array}{c}60 \\
41-76\end{array}$} \\
\hline Performance status & No. of patients & $\%$ & No. of patients & $\%$ \\
\hline 0 & 39 & 76.5 & 28 & 57.1 \\
\hline 1 & 11 & 21.6 & 21 & 42.9 \\
\hline 2 & I & 1.9 & 0 & 0 \\
\hline \multicolumn{5}{|l|}{ FIGO stage } \\
\hline lc & 4 & 7.8 & 0 & 0 \\
\hline$\|$ & 7 & 13.7 & 8 & 16.3 \\
\hline III & 37 & 72.6 & 36 & 73.5 \\
\hline IV & 3 & 5.9 & 5 & 10.2 \\
\hline \multicolumn{5}{|l|}{ Residual disease } \\
\hline None/micro & 13 & 25.5 & 13 & 26.5 \\
\hline$\leqslant 2 \mathrm{~cm}$ & 17 & 33.3 & 15 & 30.6 \\
\hline$>2 \mathrm{~cm}$ & 21 & 41.2 & 21 & 42.9 \\
\hline \multicolumn{5}{|l|}{ Histological subtype } \\
\hline Serous/papillary & 24 & 47.1 & 28 & 57.1 \\
\hline Mucinous & 4 & 7.8 & 2 & 4.1 \\
\hline Clear cell & 3 & 5.9 & 3 & 6.1 \\
\hline Endometrioid & 5 & 9.8 & 5 & 10.2 \\
\hline Other & 12 & 23.5 & 9 & 18.3 \\
\hline Not known & 3 & 5.9 & 2 & 4.1 \\
\hline
\end{tabular}

$\mathrm{FIGO}=$ International Federation of Gynecologic Oncology.

Demographic characteristics were similar in both groups (Table 2). Most (81\%) patients had FIGO stage III-IV disease and $42 \%$ had bulky residual disease ( $>2 \mathrm{~cm}$ diameter) after surgery. The most common histological subtype was serous adenocarcinoma and $64 \%$ of tumours were moderately or poorly differentiated. Although more patients in arm A were of performance status 0 , this difference was not statistically significant $\left(\chi^{2}=3.39\right.$, $P=0.066)$. Two patients who commenced therapy were subsequently withdrawn owing to incorrect histological diagnosis (one mixed mullerian tumour, one metastatic carcinoid). These patients were included in the toxicity and dose intensity analyses, but excluded from efficacy assessments.

\section{Chemotherapy administration and dose intensity}

A summary diagram indicating the progress of the two patient cohorts through treatment is given in Figure 1.

All patients received $\geqslant 1$ cycle of carboplatin AUC 7 and 88 out of 100 patients completed carboplatin therapy. Seventy-seven of 379 carboplatin cycles $(20.3 \%)$ were delayed by $\geqslant 1$ week and 27 cycles were subsequently given at reduced dose. The majority $(87 \%)$ of the delays were due to haematological toxicity. The median carboplatin dose intensity achieved was $91.7 \%$ of the planned dose in arm A and $85.9 \%$ in arm B.

Forty-three patients $(84 \%)$ in arm A commenced docetaxel chemotherapy and 36 (71\%; 90\% confidence interval (CI) $58-81 \%$ ) completed all planned therapy (primary end point). Nineteen initial cycles of docetaxel were delayed owing to carboplatininduced haematological toxicity. In contrast, only six subsequent cycles were delayed by $\geqslant 1$ week and, although 14 cycles were dose-reduced (primarily owing to neutropenic fever), the mediandose intensity achieved was $97.7 \%$. Thirty-eight patients $(78 \%)$ in arm B commenced docetaxel-irinotecan treatment and 33 (67\%; $90 \%$ CI 55-78\%) completed planned therapy (primary end point). Fifteen initial cycles were delayed owing to carboplatin-related toxicity. Twelve subsequent cycles were delayed and 17 given at reduced dose (overall, nine cycles were dose-reduced owing to neutropenic fever and seven owing to gastrointestinal toxicity (primarily diarrhoea)). Of the planned dose, the median-dose intensity was $92.6 \%$ for docetaxel and $92.4 \%$ for irinotecan. 


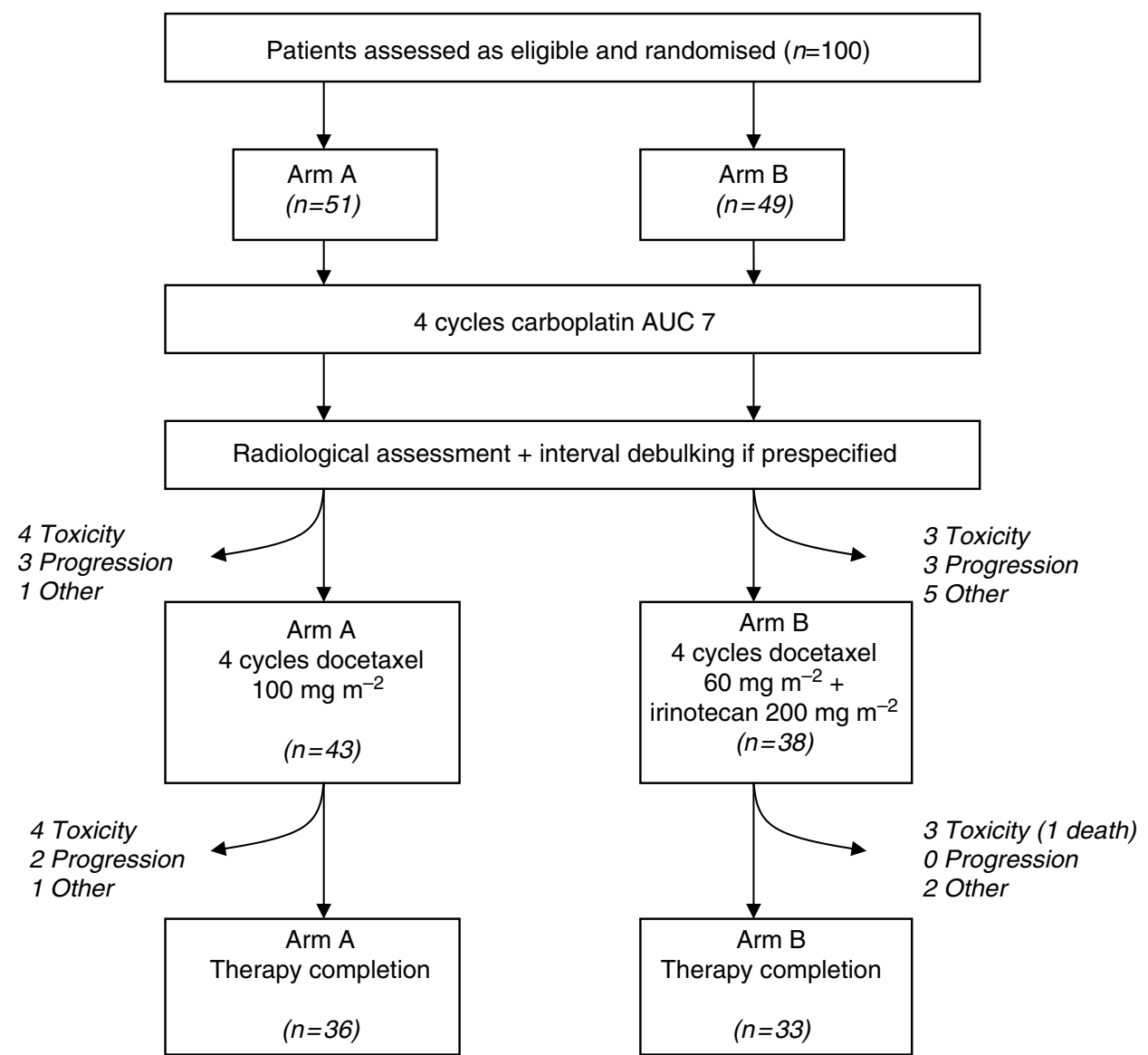

Figure I Flow chart indicating progress of the two patient cohorts through Arms A and B. All chemotherapy cycles were administered at 3-weekly intervals. Numbers of patients $(n)$ at each stage of therapy are listed. Reasons for discontinuation are given in italics.

Table 3 Percentage of cycles of chemotherapy complicated by toxicity

\begin{tabular}{|c|c|c|c|c|c|c|}
\hline \multirow{4}{*}{$\begin{array}{l}\text { No. of cycles } \\
\text { Grade }\end{array}$} & & & \multicolumn{4}{|c|}{ Docetaxel \pm irinotecan } \\
\hline & \multicolumn{2}{|c|}{ Carboplatin } & \multicolumn{2}{|c|}{ Arm A } & \multicolumn{2}{|c|}{ Arm B } \\
\hline & \multicolumn{2}{|c|}{379} & \multicolumn{2}{|c|}{161} & \multicolumn{2}{|c|}{141} \\
\hline & 3 & 4 & 3 & 4 & 3 & 4 \\
\hline \multicolumn{7}{|l|}{ Haematological toxicity } \\
\hline Neutropenia & 8.6 & 2.2 & 22.3 & 41.2 & 25.2 & 25.0 \\
\hline Anaemia & 4.1 & 0.5 & 0 & 0.7 & 0 & 0 \\
\hline Thrombocytopenia & 5.5 & 2.7 & 0 & 0 & 0.8 & 0.8 \\
\hline \multicolumn{7}{|l|}{ Gastrointestinal } \\
\hline Nausea & 1.1 & - & 0.6 & - & 2.8 & - \\
\hline Vomiting & 4.7 & 1.1 & 1.9 & 0 & 1.4 & 0 \\
\hline Diarrhoea & 1.1 & 0.3 & 0.6 & 0 & 2.8 & 0.7 \\
\hline Constipation & 0.8 & 0 & 0 & 0 & 0.7 & 0 \\
\hline Abdominal pain & 1.3 & 0 & 0 & 0 & 0.7 & 0.7 \\
\hline \multicolumn{7}{|l|}{ Neurological } \\
\hline Sensory & 0.3 & 0 & 1.9 & 0 & 0 & 0 \\
\hline Motor & 0 & 0 & 0 & 0 & 0 & 0 \\
\hline \multicolumn{7}{|l|}{ Other } \\
\hline Oedema & 0.3 & 0 & 0 & 0 & 0 & 0 \\
\hline Dyspnoea & 0.3 & 0.3 & 0 & 0 & 0 & 0 \\
\hline Stomatitis & 0.5 & 0 & 0.6 & 0 & 0 & 0 \\
\hline
\end{tabular}

Neither arm demonstrated a treatment completion rate statistically greater than $60 \%$, which was the predefined feasibility criterion.

\section{Toxicity}

The predominant toxicities reported were haematological (Table 3). As no significant toxicity differences were noted between arms A and $\mathrm{B}$ during the carboplatin treatment phase, these data were combined: $10.8 \%$ of carboplatin cycles were complicated by grade 3-4 neutropenia and $8.2 \%$ by grade 3-4 thrombocytopenia. However, only $2.1 \%$ of cycles were complicated by neutropenic fever and $1.1 \%$ by thrombocytopenia-related bleeding. Apart from vomiting (grade 3-4 in $5.8 \%$ of cycles), non-haematological toxicities were generally mild throughout carboplatin treatment.

During the docetaxel-based treatment phase, differences in toxicity profile were evident between the two arms. Grade 3-4 neutropenia was reported in 63.5 and $50.2 \%$ of cycles in arms A and $\mathrm{B}$, respectively; neutropenic fever complicated 7.5 and $5.7 \%$ of cycles, respectively. The incidences of other haematological toxicities were low in both arms. Gastrointestinal toxicity was more common in arm B, with grade 3-4 diarrhoea seen in $3.5 \%$ of cycles compared with only $0.6 \%$ in arm A. Sensory neurotoxicity was reported more frequently in arm $\mathrm{A}$, consistent with the higher docetaxel dose; $5.0 \%$ of cycles in arm A vs $1.4 \%$ in arm B were complicated by grade $2-4$ sensory neuropathy (1.9 vs $0 \%$ by grade 3-4 sensory neuropathy). The differences in toxicity were reflected in the reasons for dose reduction or delay noted above. Grade 2 alopecia was reported in 43.7 and $50.4 \%$ of cycles in arms A and B, respectively. Overall, 14 patients (eight in arm A, six in arm B) discontinued protocol treatment because of toxicity; the reasons are listed in Table 4.

Two deaths due to adverse events were reported (both in arm B): one patient died from enterococcal septicaemia and pneumonia 
Table 4 Reasons for treatment discontinuation

\begin{tabular}{|c|c|c|c|c|c|c|c|c|c|c|}
\hline \multirow[b]{2}{*}{ Cycle } & \multicolumn{5}{|c|}{ Arm A } & \multicolumn{5}{|c|}{ Arm B } \\
\hline & $\begin{array}{l}\text { No. of patients } \\
\text { completed }\end{array}$ & Toxicity & Progression & Consent & Other & $\begin{array}{l}\text { No. of patients } \\
\text { completed }\end{array}$ & Toxicity & Progression & Consent & Other \\
\hline 1 & 51 & - & - & - & - & 49 & - & - & - & - \\
\hline 2 & 49 & 1 & I & 0 & 0 & 48 & I & 0 & 0 & 0 \\
\hline 3 & 48 & 2 & 1 & 0 & 0 & 46 & 2 & I & 0 & 0 \\
\hline 4 & 45 & 4 & 2 & 0 & 0 & 43 & 3 & 2 & I & 0 \\
\hline 5 & 43 & 4 & 3 & 1 & 0 & 38 & 3 & 3 & 2 & 3 \\
\hline 6 & 42 & 5 & 3 & 1 & 0 & 35 & 4 & 3 & 4 & 3 \\
\hline 7 & 40 & 5 & 5 & I & 0 & 35 & 4 & 3 & 4 & 3 \\
\hline 8 & 36 & 8 & 5 & 1 & I & 33 & 6 & 3 & 4 & 3 \\
\hline
\end{tabular}

associated with grade 4 neutropenia 5 days after receiving docetaxel and irinotecan and one patient died from secondary complications due to a treatment-unrelated ischaemic stroke suffered during the carboplatin treatment phase. At the time of death, this latter patient had discontinued protocol treatment owing to disease progression.

\section{Efficacy}

At baseline, 30 patients in arm $\mathrm{A}$ and 25 in $\operatorname{arm} \mathrm{B}$ had radiologically evaluable disease. Of these, 17 (57\%) in arm A achieved a disease response after the carboplatin phase, compared with $12(48 \%)$ in arm B (Table 5). After completion of docetaxelbased treatment, seven patients $(23 \%)$ in arm A had achieved a complete response and eight $(27 \%)$ had achieved a partial response; seven $(23 \%)$ patients had disease progression. In arm B, two patients (8\%) achieved a complete response and $10(40 \%)$ achieved a partial response; seven (28\%) patients had disease progression.

The change in cytotoxic agents after the first four cycles allowed a preliminary assessment of the relative efficacies of docetaxel $v s$ docetaxel-irinotecan in improving disease status. Of the 22 patients in arm A and 19 patients in arm B with partial response or stable disease after carboplatin initiation therapy, six (27\%) had improved radiological findings after docetaxel (arm A) compared with three (16\%) after docetaxel-irinotecan (arm B) (odds ratio $=0.5 ; 95 \%$ CI $0.147-1.702)$. Disease progression was seen in three $(14 \%)$ docetaxel patients and three (16\%) docetaxelirinotecan patients.

Although nine patients (9\%) had disease progression after carboplatin, only three (3\%) remained on protocol. Only one of these patients (arm B) responded to further therapy. Of the patients with radiologically unevaluable disease at trial entry, two patients (one in each arm) experienced disease progression during docetaxel-based therapy.

Sixty-one patients $(61 \%)$ had disease that could be assessed for CA-125 response using the Gynecologic Cancer Intergroup (GCIG) criteria (Rustin et al, 2004). Forty-one of these patients (67\%) met the criteria for response, 33 of whom had radiologically measurable disease. Of these patients, 27 had a radiological disease response, five had stable disease and one had radiological disease progression. Of the patients whose CA-125 failed to respond to therapy and who had radiologically measurable disease (11 patients), three had a disease response on cross-sectional imaging.

The median follow-up period was 17.1 months, during which time 49 patients (50\%) experienced disease progression. Median progression-free survival was 17.1 months in arm A (95\% CI 11.7 22.4 months) and 15.9 months in arm B (95\% CI 8.0-23.9 months)
Table 5 Radiological response assessment in patients with evaluable disease at trial entry

\begin{tabular}{|c|c|c|c|c|}
\hline & \multicolumn{2}{|c|}{ Arm A $(n=30)$} & \multicolumn{2}{|c|}{ Arm B $(n=25)$} \\
\hline & Post-carboplatin & Overall & Post-carboplatin & Overall \\
\hline$C R$ & $3(10)$ & $7(23)$ & $0(0)$ & $2(8)$ \\
\hline PR & $14(47)$ & $8(27)$ & $12(48)$ & $10(40)$ \\
\hline$C R+P R$ & $17(57)$ & $15(50)$ & $12(48)$ & $12(48)$ \\
\hline SD & $8(27)$ & $5(17)$ & $7(28)$ & $4(16)$ \\
\hline PD & $4(13)$ & 7 (23) & $5(20)$ & $7(28)$ \\
\hline Measurable disease ${ }^{a}$ & I (3) & $3(10)$ & I (4) & $2(8)$ \\
\hline Non-evaluable & \multicolumn{2}{|c|}{$20(-)$} & \multicolumn{2}{|c|}{$23(-)$} \\
\hline
\end{tabular}

a Failed to complete therapy. Values in parentheses are percentages given as the proportion of patients with radiologically evaluable disease at trial entry. $\mathrm{CR}=$ complete response; $\mathrm{PD}=$ progressive disease; $\mathrm{PR}=$ partial response; $\mathrm{SD}=$ stable disease

\section{Quality of life}

Of the eligible patients, $82 \%$ completed the QoL questionnaires at baseline (77\% after four cycles and $71 \%$ after eight cycles). However, only $42 \%$ of eligible patients completed questionnaires at the first post-treatment follow-up. No significant differences were seen between the two arms in overall change in QoL or change in any symptom-specific subscales.

\section{DISCUSSION}

This randomised phase II study assessed the feasibility of delivering sequential schedules of carboplatin, docetaxel and irinotecan as first-line therapy in advanced EOC. One hundred patients were randomised to receive four 3-weekly cycles of either docetaxel $100 \mathrm{mg} \mathrm{m}^{-2}$ (arm A) or docetaxel $60 \mathrm{mg} \mathrm{m}^{-2}$ and irinotecan $200 \mathrm{mg} \mathrm{m}^{-2}$ (arm B) after four cycles of carboplatin at a dose of AUC 7 every 3 weeks. Both arms just failed to meet the predefined feasibility criterion of a treatment completion rate statistically greater than $60 \%$ (arm A $71 \%$, 90\% CI $58-81 \%$, $P=0.079$; arm B $67 \%, 90 \%$ CI $55-78 \%, P=0.184)$.

The overall response rates achieved in patients with radiologically evaluable disease of $50 \%$ in arm A and $48 \%$ in arm B appear modest for a phase II study in this patient group, despite a median dose intensity of $>85 \%$ for all drugs administered. The results, however, are consistent with the $58.7 \%$ achieved with carboplatindocetaxel in the SCOTROC I trial on which this study was based (Vasey et al, 2004). Importantly, the median progression-free survival figures of 17.1 and 15.9 months in arms A and B, respectively, are also similar to those previously reported in phase II and III studies of first-line chemotherapy in patients with 
predominantly stage III-IV EOC (International Collaborative Ovarian Neoplasm Group, 2002; Guppy et al, 2004; Harries et al, 2004; Vasey et al, 2004), providing preliminary evidence that the regimens we tested have efficacy consistent with current first-line therapies.

The use of sequential therapy also allowed a preliminary assessment of the relative efficacies of docetaxel and docetaxelirinotecan in improving disease status after single-agent carboplatin. Patients with persistent disease after carboplatin treatment represented a cohort of patients in this trial with relatively chemoresistant disease. In this cohort, $27 \%$ of patients receiving docetaxel alone had improved disease status compared with $16 \%$ of patients receiving docetaxel-irinotecan (odds ratio $=0.5 ; 95 \%$ CI 0.147-1.702). These figures are consistent with equivalence between the two treatment arms in a study of this size (Fisher's exact test $P=0.62$ ). They are, however, lower than the response rates reported in a Finnish phase II study of docetaxel-irinotecan in relapsed EOC, which documented a response rate of $63 \%$ in both platinum-sensitive and refractory disease (Mäenpää et al, 2002). However, Guppy et al (2004) reported decreasing response rates for each agent in their small phase II study using sequential carboplatin, paclitaxel and topotecan in a similar patient group to that investigated here.

The present study did reveal differences in the toxicity profiles of the two treatment regimens under investigation, although the toxicities seen were, in general, predictable and manageable. Myelosuppression was the most frequent toxicity $(10.8 \%$ of carboplatin cycles, $63.5 \%$ of docetaxel cycles and $50.2 \%$ of docetaxel-irinotecan cycles were associated with grade 3-4 neutropenia), although the rates of complicated neutropenia were low. Differences were also seen with non-haematological toxicity. Significant sensory neuropathy was more common with docetaxel alone (probably secondary to the higher docetaxel dose) whereas serious gastrointestinal side effects were more frequent with docetaxel-irinotecan. A total of $3.5 \%$ of cycles of combination chemotherapy were complicated by grade III/IV diarrhoea compared with $0.9 \%$ of those using docetaxel alone, and one patient in arm B died of enterococcal septicaemia. While this toxicity pattern has been reported in other phase II studies of docetaxel-irinotecan combinations, it is generally considered acceptable and is not affected by regimen scheduling (Kurtz et al, 2003; Pectasides et al, 2005; Wachters et al, 2005). While such differences were apparent using conventional toxicity scores, no significant QoL differences emerged (either on global measures or on the symptom-specific subscales). Although the EORTC-QLQ30 score has previously been reported to correlate well with toxicity scores for ovarian cancer patients undergoing chemotherapy (Butler et al, 2004), the low number of severe objective toxicities and the relatively poor compliance with questionnaire completion may explain the inability of this modality to detect differences in this study.

During the carboplatin initiation phase, seven patients (7\%) withdrew owing to excessive toxicity and, subsequently, four patients $(9.3 \%)$ receiving docetaxel and three $(7.9 \%)$ receiving docetaxel-irinotecan discontinued therapy owing to toxicity, giving a total withdrawal rate of $14 \%$. These figures compare favourably with those seen in a phase II study of the triplet combination carboplatin AUC 5/paclitaxel $150 \mathrm{mg} \mathrm{m}^{-2}$ /irinotecan $100 \mathrm{mg} \mathrm{m}^{-2}$ administered every $3-4$ weeks, where $27 \%$ of patients withdrew owing to toxicity (Escobar et al, 2004).

Most previous studies investigating the incorporation of topoisomerase-I inhibitors into first-line therapy of EOC have used triplet combinations, with subsequent difficulties in dose escalation owing to myelotoxicity (Herben et al, 1999; Cacciari et al, 2000; Bolis et al, 2001; Escobar et al, 2004). The results reported here indicate the feasibility of this concept by using a sequential approach to maintain dose intensity. In particular, a similar total dose of carboplatin (AUC $28 v s 30$ ) was administered compared to standard carboplatin-paclitaxel regimens and a higher dose intensity (AUC 2.5 vs 1.25 per week) was achieved during the platinum phase of therapy. We also documented a manageable toxicity profile for docetaxel-irinotecan that reduces neurotoxicity compared to using a higher dose of docetaxel alone although at the expense of increased diarrhoea. However, the recent results of two randomised phase III studies (De Placido et al, 2004; Pfisterer et al, 2005) have failed to demonstrate an improvement in survival with the addition of four cycles of the topoisomerase-I inhibitor topotecan after standard carboplatinpaclitaxel as first-line treatment for EOC. Although the MITO-1 study (De Placido et al, 2004) can be criticised for only effectively assessing the concept of consolidation therapy, as $87 \%$ of patients randomised to topotecan or placebo had already obtained a complete response to standard treatment, patients were randomised at registration in the GINECO/AGO-OVAR trial (Pfisterer et al, 2005) and $78 \%$ of those randomised to sequential topotecan received this therapy.

Nevertheless, the present study suggests that further investigation of the concept of delivering sequential combinations of carboplatin, docetaxel and irinotecan at high-dose intensity to patients with ovarian cancer may be appropriate.

\section{ACKNOWLEDGEMENTS}

We thank Mia Parkinson and Joseph Cope for data management and Rick Swindell and Mark Dougal (Department of Medical Statistics, Christie Hospital, Manchester, UK) for their invaluable help in providing statistical analysis of the trial data. We acknowledge all the participating clinicians who recruited patients. Finally, we thank Professor Gordon Rustin and Gavin Shreeve (Department of Medical Oncology, The Clocktower, Mount Vernon Hospital, Northwood, UK) for their help in analysing the CA-125 response data. This research was supported in part by grants from Aventis Pharmaceuticals Inc. (Bridgewater, NJ, USA).

\section{REFERENCES}

Bodurka DC, Levenback C, Wolf JK, Gano J, Wharton JT, Kavanagh JJ, Gershenson DM (2003) Phase II trial of irinotecan in patients with metastatic epithelial ovarian cancer or peritoneal cancer. J Clin Oncol 21: $291-297$

Bolis G, Scarfone G, Villa A, Parazzini F (2001) Phase II trial of topotecan, carboplatin and paclitaxel as front-line therapy in suboptimal advanced epithelial ovarian cancer. Gynecol Oncol 81: $331-333$

Bonadonna G, Zambetti M, Valagussa P (1995) Sequential or alternating doxorubicin and CMF regimens in breast cancer with more than three positive nodes. Ten-year results. JAMA 273: 542-547

Butler L, Bacon M, Carey M, Zee B, Tu D, Bezjak A (2004) Determining the relationship between toxicity and quality of life in an ovarian cancer chemotherapy clinical trial. J Clin Oncol 22: 2461-2468

Cacciari N, Zamagni C, Martoni A (2000) The addition of topotecan to carboplatin and paclitaxel as first-line therapy for advanced ovarian cancer: is it possible only with peripheral blood stem support? Eur J Gynaecol Oncol 21: $84-85$

Calvert AH, Newell DR, Gumbrell LA, O’Reilly S, Burnell M, Boxall FE, Siddik ZH, Judson IR, Gore ME, Wiltshaw E (1989) Carboplatin dosage: prospective evaluation of a simple formula based on renal function. J Clin Oncol 7: $1748-1756$ 
De Placido S, Scambia G, Di Vagno G, Naglieri E, Lombardi AV, Biamonte R, Marinaccio M, Carteni G, Manzione L, Febbraro A, de Matteis A, Gasparini G, Valerio MR, Danese S, Perrone F, Lauria R, De Laurentiis M, Greggi S, Gallo C, Pignata S (2004) Topotecan compared with no therapy after response to surgery and carboplatin/paclitaxel in patients with ovarian cancer: Multicenter Italian Trials in Ovarian Cancer (MITO-1) randomized study. J Clin Oncol 22: 2635-2642

Escobar P, Markman M, Rose P, Zanotti K, Webster K, Belinson J (2004) Phase 2 trial of carboplatin, paclitaxel and irinotecan in ovarian, fallopian tube and primary peritoneal cancers. Gynecol Oncol 92: $192-196$

Fleming TR (1982) One-sample multiple testing procedure for phase II clinical trials. Biometrics 38: $143-151$

Gordon AN, Fleagle JT, Guthrie D, Parkin DE, Gore ME, Lacave AJ (2001) Recurrent epithelial ovarian carcinoma: a randomized phase III study of pegylated liposomal doxorubicin versus topotecan. J Clin Oncol 19: $3312-3322$

Guastalla III JP, Dieras V (2003) The taxanes: toxicity and quality of life considerations in advanced ovarian cancer. Br J Cancer 89(Suppl 3): S16-S22

Guppy AE, Nelstrop AE, Foster T, Agarwal R, Seckl MJ, Rustin GJS (2004) A phase II study of sequential carboplatin, paclitaxel and topotecan in patients with previously untreated advanced ovarian cancer. $\mathrm{Br}$ J Cancer 90: $810-814$

Harries M, Moss C, Perren T, Gore M, Hall G, Everard M, A'Hern R, Gibbens I, Jenkins A, Shah R, Cole C, Pizzada O, Kaye S (2004) A phase II feasibility study of carboplatin followed by sequential weekly paclitaxel and gemcitabine as first-line treatment for ovarian cancer. Br J Cancer 91: $627-632$

Herben VM, Panday VR, Richel DJ, Schellens JH, van der Vange N, Rosing $\mathrm{H}$, Beusenberg FD, Hearn S, Doyle E, Beijnen JH, ten Bokkel Huinink WW (1999) Phase I and pharmacologic study of the combination of paclitaxel, cisplatin and topotecan administered intravenously every 21 days as first-line therapy in patients with advanced ovarian cancer. J Clin Oncol 17: $747-755$

International Collaborative Ovarian Neoplasm Group (2002) Paclitaxel plus carboplatin versus standard chemotherapy with either single-agent carboplatin or cyclophosphamide, doxorubicin and cisplatin in women with ovarian cancer: the ICON3 randomised trial. Lancet 360: 505-515

Kurtz JE, Negrier S, Husseini F, Limacher JM, Borel C, Wagner JP, Prevot G, Bergerat JP, Dufour P (2003) A phase II study of docetaxel-irinotecan combination in advanced pancreatic cancer. Hepatogastroenterology 50: $567-570$

Mäenpää J, Ala-Fossi S-L, Kivinen S, Pohto M, Käär K, Jehunen A (2002) Docetaxel and irinotecan in the second-line treatment of ovarian cancer: final results of a phase II study. Proc Am Soc Clin Oncol 18: 403 (Abstract)

Mäenpää J, Hagman E, Kivinen S, Pohto M, Käär K, Jekunen A (1998) The combination of taxotere and campto for second line treatment of ovarian cancer - a phase I dose finding study. Ann Oncol 9(Suppl 4): 69 (Abstract)
McGuire WP, Hoskins WJ, Brady MF, Kucera PR, Partridge EE, Look KY, Clarke-Pearson DL, Davidson M (1996) Cyclophosphamide and cisplatin compared with paclitaxel and cisplatin in patients with stage III and stage IV ovarian cancer. $N$ Engl J Med 334: 1-6

Muggia FM, Braly PS, Brady MF, Sutton G, Niemann TH, Lentz SL, Alvarez RD, Kucera PR, Small JM (2000) Phase III randomized study of cisplatin versus paclitaxel versus cisplatin and paclitaxel in patients with suboptimal stage III or IV ovarian cancer: a Gynecologic Oncology Group study. J Clin Oncol 18: 106-115

Pectasides D, Pectasides M, Farmakis D, Kostopoulou V, Nikolaou M, Gaglia A, Koumppou M, Mylonakis N, Xiros N, Economopoulos T, Raptis SA (2005) Comparison of docetaxel and docetaxel-irinotecan combination as second-line chemotherapy in advanced non-small-cell lung cancer: a randomized phase II trial. Ann Oncol 16: 294-299

Pfisterer J, Weber B, du Bois A, Lortholary A, Schade-Brittinger C, Wagner U, Bourgeois H, Jakisch C, Moebus V, Mayer F (2005) Paclitaxel/ carboplatin $v s$. paclitaxel/carboplatin followed by topotecan in first-line treatment of advanced ovarian cancer. Mature results of a gynecologic cancer intergroup phase III trial of the AGO OVAR and GINECO. J Clin Oncol 23(16S): 5007

Piccart M, Bertelsen K, James K, Cassidy J, Mangioni C, Simonsen E, Stuart G, Kaye S, Vergote I, Blom R, Grimshaw R, Atkinson RJ, Swenerton KD, Trope C, Nardi M, Kaern J, Tumolo S, Timmers P, Roy JA, Lhoas F, Lindvall B, Bacon M, Birt A, Andersen JE, Zee B, Paul J, Baron B, Pecorelli S (2000) Randomized intergroup trial of cisplatin-paclitaxel versus cisplatin-cyclophosphamide in women with advanced epithelial ovarian cancer: three-year results. J Natl Cancer Inst 92: 699-708

Rustin GJ, Quinn M, Thigpen T, du Bois A, Pujade-Lauraine E, Jakobsen A, Eisenhauer E, Sagae S, Greven K, Vergote I, Cervantes A, Vermorken J (2004) New guidelines to evaluate the response to treatment in solid tumors (ovarian cancer). J Natl Cancer Inst 96: 487-488

Shibuya K, Mathers CD, Boschi-Pinto C, Lopez AD, Murray CJ (2002) Global and regional estimates of cancer mortality and incidence by site: II. Results for the global burden of disease 2000. BMC Cancer 2: 37

Takeuchi S, Dobashi K, Fujimoto S, Tanaka K, Suzuki M, Terashima Y, Hasumi K, Akiya K, Negishi Y, Tamaya T (1991) A late phase II study of CPT-11 on uterine cervical cancer and ovarian cancer. Research Groups of CPT-11 in Gynecologic Cancers. Gan To Kagaku Ryoho 18: $1681-1689$

Vasey PA, Jayson GC, Gordon A, Gabra H, Coleman R, Atkinson R, Parkin D, Paul J, Hay A, Kaye SB, the Scottish Gynaecological Cancer Trials Group (2004) Phase III randomized trial of docetaxel-carboplatin versus paclitaxel-carboplatin as first-line chemotherapy for ovarian carcinoma. I Natl Cancer Inst 96: $1682-1691$

Wachters FM, Groen HJM, Biesma B, Schramel FMNH, Postmus PE, Stigt JA, Smit EF (2005) A randomised phase II trial of docetaxel vs docetaxel-irinotecan in patients with stage IIIb-IV non-small-cell lung cancer who failed first-line treatment. Br J Cancer 92: 15-20

Wahl AF, Donaldson KL, Fairchild C, Lee FY, Foster SA, Demers GW, Galloway DA (1996) Loss of normal p53 function confers sensitization to Taxol by increasing G2/M arrest and apoptosis. Nat Med 2: $72-79$ 